\title{
Féeries
}

Études sur le conte merveilleuX, XVII $-\mathrm{XIX}{ }^{\mathrm{e}}$ siècle

\section{Marie-Louise Tenèze, Les Contes merveilleux français. Recherche de leurs organisations}

\section{narratives}

Paris, Maisonneuve \& Larose, 2004, 164 p.

\section{Christian Abry}

\section{CpenEdition}

Journals

Édition électronique

URL : http://journals.openedition.org/feeries/178

DOI : $10.4000 /$ feeries. 178

ISSN : 1957-7753

Éditeur

UGA Éditions/Université Grenoble Alpes

Édition imprimée

Date de publication : 1 février 2006

Pagination : 393-397

ISBN : 2-84310-082-8

ISSN : $1766-2842$

\section{Référence électronique}

Christian Abry, « Marie-Louise Tenèze, Les Contes merveilleux français. Recherche de leurs

organisations narratives », Féeries [En ligne], 3 | 2006, mis en ligne le 04 mai 2007, consulté le 23

septembre 2020. URL : http://journals.openedition.org/feeries/178 ; DOI : https://doi.org/10.4000/

feeries. 178

Ce document a été généré automatiquement le 23 septembre 2020.

(c) Féeries 


\title{
Marie-Louise Tenèze, Les Contes merveilleux français. Recherche de leurs organisations narratives
}

Paris, Maisonneuve \& Larose, 2004, 164 p.

\author{
Christian Abry
}

Mauvaise nouvelle pour ceux qui détestent le catalogage (ils sont nombreux - et pas qu'en France - dans la communauté des chercheurs sur le conte!): Marie-Louise Tenèze vient d'opérer depuis sa retraite la jonction entre Propp et le catalogue français, le Delarue-Tenèze (continué par Josiane Bru). Cette même année 2004 l'international Aarne-Thompson vient de se voir considérablement augmenté, et pour ceux qui continueront à juger utile de faire référence aux œuvres du patrimoine narratif de l'Humanité, il faudra sigler dorénavant les types ATU, additionnant ainsi le mérite de Hans-Jörg Uther qui vient de réaliser cette prouesse depuis l'Enzyklopädie des Märchens $(E M)$ à Göttingen. D'autres bonnes nouvelles sont venues des antiquistes: après G. Anderson, Fairytale in the Ancien World, Routledge, 2000 (c.r. de F. Létoublon in Gaia, 2005, p. 195-199), on possède maintenant un Ariadne's Thread. A Guide to International Tales Found in Classical Literature, Cornell University Press, 2002, grâce à W. Hansen. Estce à dire que tout s'illumine dans le repérage du ciel des narrations ? Claude Brémond continue dans sa logique du récit, notamment pour l'établissement de l'Index des passions, actions et motivations dans les Mille et Une Nuits (avec Aboubakr Chraïbi). Au-delà de la typologie du conte, les actes des rencontres de 2003 et 2004 «Indexer les exempla médiévaux » (parues dans les Cahiers du Centre de recherches historiques, $n^{\circ} 35$, avril 2005, p. 9-81) ne font pas véritablement acte d'enthousiasme. Quant aux types des récits légendaires (Sagen), l'entreprise juste initiée en 1958 par R.T. Christiansen, The Migratory Legends ( FFC $\mathrm{n}^{\circ} 175$, Helsinki, $2^{\text {nde }}$ éd. 1992), n'a guère suscité depuis de vocations. Il en va de même pour les unités de taille variable plus petites (voire égales au type): le monumental Motif-Index de Thompson (2 nd éd. 1966, avec version électronique disponible) s'est certes attiré, encore dernièrement au tournant de ce xxie siècle, des recommandations bien documentées pour sa continuation (H. Jason, Motif, 
Type and Genre. A Manual for Compilation of Indices \& A Bibliography of Indices and Indexing, FFC $\mathrm{n}^{\circ} 273$, Helsinki, 2000). À notre connaissance, les deux dernières meilleures surprises pour leurs indexations, qui continuent et étendent l'excellent et sous-utilisé E.W. Baughman, Type and Motif-Index of the folktales [and belief legends] of England and North America, Bloomington, 1966, complément indispensable au Motif-Index, auront été réalisées : à l'autre bout du monde, pour les récits de Nouvelle-Guinée parus dans le Wantok Newspaper, traduits du pidgin par T.H. Slone, One thousand one Papua New Guinean nights, Oakland, Masalai Press, 2001; et ici-même, par la sortie du premier tome de l'édition préparée par N. Abry et A. Joisten de la collecte de C. Joisten, Êtres fantastiques du Dauphiné. Patrimoine narratif de l'Isère, Grenoble, Musée dauphinois, 2005.

C'est dans ce contexte que M.-L. Ténèze nous fait la surprise de ce petit livre dense, à l'exposé concis et ramassé, fruit de toute une vie d'expérience dans l'analyse du récit au sein de l'entreprise du catalogage. "Dans l'espoir que ce dernier-né ne vous paraîtra pas trop rébarbatif » m'écrit-elle sur le carton de ce cadeau... Je n'irai pas me vanter d'avoir été touché d'être toujours présent dans les attentions de la Dame du conte merveilleux français. Elle sait bien que ce que je tiens du conte, je le tiens de Charles et Alice Joisten et de leurs amis et connaissances. Ce peu de bagage personnel et ce lourd lest de mes amis à qui je ne réclame aucun répit de test, me libère de l'utopique tâche unificatrice d'ancien fondateur de l'Association des étudiants en linguistique et poétique, née à Grenoble en 1968, ayant viré ethnographe de la parole narrative, tout en restant linguiste phonéticien profondément ancré dans le contrôle de la parole articulatoire. Ce que d'autres ont peu réussi (même en psychologie cognitive de la narration) depuis le structuralisme - méprisant Antti Aarne et Stith Thompson, et ne comprenant même pas que Propp puisse trouver à redire à la vision que s'en donna Lévi-Strauss (sans parler de l'incompréhension des Racines historiques du conte merveilleux et des prolongements réalisés par Meletinski) - M.-L. Ténèze le tente et le résultat de cette tenace persévérance d'expérience a de quoi couper le souffle de plus d'un (pr) édicteur théoricien.

$3 \mathrm{Au}$ début de sa conclusion intitulée "Constatations et Perspectives » M.-L. Ténèze rappelle: «Dans mon souci [...] de ne pas «rabattre» le genre sur sa prétendue "formule canonique", $\mathrm{j}$ 'ai pris au contraire très pragmatiquement appui sur la diversité des contes-types telle que l'a mise en évidence le Catalogue» (p. 153). Et de citer en fin cet aveu de Lévi-Strauss lui-même (p. 158) : «Chez Propp le formalisme aboutit à la découverte qu'il n'existe en réalité qu'un seul conte. Dès lors... nous savons ce qu'est le conte, mais comme l'observation nous met en présence, non pas d'un conte archétypal, mais d'une multitude de contes particuliers, nous ne savons plus comment les classer. Avant le formalisme, nous ignorions sans doute ce que ces contes avaient en commun. Après lui, nous sommes privés de tout moyen de comprendre en quoi ils diffèrent. On a bien passé du concret à l'abstrait, mais on ne peut plus redescendre de l'abstrait au concret. » (Anthropologie structurale deux, Paris, Plon, 1973, p. 139-173).

4 Nous n'allons pas reprendre ici cette analyse fine et cohérente des mouvements (cf. Propp) narratifs des contes merveilleux français. D'entrée, une «conception plus abstraite, plus libre du mouvement me conduit nécessairement à prendre de la hauteur par rapport à la description tant actantielle que fonctionnelle de la Morphologie.» (p. 8 ; réflexion reprise en conclusion, p. 155 et suiv.). D'autre part l'introduction (p. 10), dans la relation Héros vs. Antagoniste/Objet, de la distinction entre opposition externe (de 
l'Autre Monde) et opposition interne (de Ce Monde) aboutit à deux figures du Manque et deux figures de la Malfaisance (ou Méfait) :

5 I. Pour les mouvements à opposition externe

I.1. Si l'Antagoniste de l'Autre Monde est lié à l'objet de ce même Autre Monde, il ne peut y avoir que Manque de l'Objet (et non Malfaisance)

I.2. Si l'Antagoniste de l'Autre Monde est lié à un Objet de Ce Monde, il y a Malfaisance sur l'objet

II. Pour les mouvements à opposition interne

II.1. S'il y a action de l'Antagoniste de Ce Monde, pour disjoindre le Héros de son Objet, il y a Malfaisance sur le Héros (" héros-victime »)

II.2. Si la disjonction est donnée d'emblée, il n'y a que Manque

6 Mouvements et types d'opposition aboutissent à l'économie tripartite de l'ouvrage en trois «Livres": structures d'opposition externe à mouvement simple; structures mixtes, avec une opposition externe, suivie d'une opposition interne; structures d'opposition interne, les unes à mouvement simple, les autres à deux oppositions internes. Le Livre quatrième est consacré aux « Analyses particulières ». À la fin comme au début les «marginalisations voire exclusions » sont clairement annoncées. Pour le trop célèbre Chaperon rouge et pour Ma mère m'a tué, renvoi est fait aux analyses parues sur la collecte Victor Smith, dans M.-L. Tenèze \& G. Delarue, Nannette Lévesque [1803-1880] conteuse et chanteuse du pays des sources de la Loire, Paris, Gallimard, 2000 (p. 102-111 et 118-124).

7 Certains diront à quoi bon cette jonction par une "analyse différenciée » entre l'hypothèse de Propp et le corpus français ? D'autres, comme moi, avanceront que nous connaissons si mal le conte que l'admiration pour l'unité de l'objet «conte merveilleux ", que nous fait partager à plusieurs reprises M.-L. Ténèze, n'est qu'une illusion devant les beautés de la Culture qui, comme la Nature, nous cache sous l'apparence d'un papillon un affreux bricolage d'ocelles biochimiquement autoémergentes, qui réussissent à effrayer les oiseaux prédateurs, des yeux-leurres sur des ailes qui ne sont avant tout que des panneaux solaires collecteurs de photons pour démarrer plus tôt un moteur à lymphe engourdi par la rosée du matin (la fonction vol ne serait qu'un sous-produit évolutif d'une fonction de thermorégulation, pour des ailes d'insectes héritées génétiquement des branchies des crustacés). D’où les quelques questions ci-dessous - que je me pose avant tout à moi-même - sur ce qui est reconnu comme la dynamique du conte merveilleux, des mouvements partant du Manque ou du Méfait, un Manque au final comblé, un Méfait réparé.

8 Pourquoi ne trouve-t-on pas de manque ou de méfait final dans le conte " de fées »? Pardi ! Parce que ce serait un récit légendaire, par l'échec même du mariage avec la fée de l'Autre monde, ou simplement un de ces êtres étranges des parages des villages que sont les fées êtres sauvages au féminin comme au masculin. Bon! Promis je ne ramène pas ce Chaperon rouge franchouillard, anomal ou incompréhensible par bien des aspects, (sans même parler de sa male fin non grimmesque), si l'on ne voyage pas entre Tyrol et Dauphiné, et c'est une autre histoire (en attendant, cf. l'article récent de C. Shojaei Kawan dans l'EM, 11, 854-868). Mais il existe des récits légendaires où l'on se débarrasse bien apotropaïquement du danger du prédateur, un récit d'avertissement à « happy end » en quelque sorte. Toujours est-il qu'il n'y a jusque-là rien de fondamental dans ces remarques, qui font simplement état de la diversité des genres narratifs et de leur variation. L'article Dynamik que rédigea pour l'EM (3, 951-957), le grand Max Lüthi, avec 
toute la finesse de traitement qui lui était habituelle, distingue bien en effet des dynamiques propres au conte, au récit légendaire, à la légende hagiographique, au mythe, au conte facétieux...

9 Les trois exemples ci-après de manque au final nous semblent cependant résister à notre questionnement.

1. En admettant avec Meletinski (Quaderni di Semantica, 15, 1, 1994, p. 79-85) que le Trickster peut être le personnage le plus archaïque de l'art verbal de l'Humanité (comme le Corbeau créateur de Sibérie et de la Côte Ouest), celui-ci fonctionne typiquement pour expliquer le manque actuel, qui résulte d'une action qui a fait foirer ce qui marchait à merveille, comme ce célèbre long pénis du Trickster Winnebago (certes avec des sous-produits «plantureux » issu de ses morceaux), comme le Paradis Terrestre, et ici dans les Alpes, comme l'Homme sauvage, tourné en dérision alors qu'il savait tirer de la cire (du miel, du sucre... de l'or) du dernier résidu du lait après les fromages.

11 2. «Little Piggie... » et ses nombreux comparses non british (cf. l'article Fingererzählungen de R. Schenda, EM, 4, 1146-1158) est sans doute l'un des premiers contes, prodigué aux tout-petits sur les doigts de la main : que le petit doigt n'ait pour finir rien du tout à manger et qu'on se moque de lui (avec chute chatouilleuse attendue), peut rester, même dans une tête d'adulte, comme le souvenir d'une injustice.

12 3. Enfin, dans les formules de clôture du conte merveilleux: on l'épousa et on fit bombance... Et le conteur? «Ils ont fait des grandes noces, des grandes gloires,/ Et moi, j'ai pas eu la moitié d'une poire» (pirouette pas rare).

13 Sous ces trois aspects, de genèse comparée (phylogénétique), développementale (ontogénétique), et dans sa maturité (sociogénétique), le récit finit par rire du manque, sous les modes propres du rire Trickster, certes. Est-ce cette fonction princeps qui, en clôture grâce au maître du récit, reprend le dessus sur l'illusion du conte merveilleux...?

Il ne me semble pas que ce type de questions ait reçu de réponses depuis que Max Lüthi rédigea cet article fondamental de l'EM, où il tire par ailleurs pleinement profit, pour le conte, de l'analyse que fit MLT du statisme du conte animalier (parue alors dans le t. 3 du Delarue-Tenèze), qu'elle contrastait déjà avec la dynamique du conte merveilleux, en précursion de ce petit opuscule qui fera tout aussi date que le grand Catalogue. 